\section{Two cases of near asphyxiation in children, using non-releasing plastic garden ties}

We read with interest the emergency casebook featuring two cases of near asphyxiation. ${ }^{1}$ It is our practice to admit all cases of near strangulation who present early with signs or symptoms in keeping with the history for a period of observation. We adopt this policy on the basis that it is possible to miss occult, significant upper airway pathology in victims of near strangulation ${ }^{2}$ and airway obstruction can present as late as 36 hours after such an event. ${ }^{3}$ In addition it is possible to overlook visual impairment in such patients as subtle changes in visual acuity may not initially be apparent. ${ }^{4}$ Cases of near asphyxiation in children are not widely reported in the literature and therefore it is difficult to have an evidence based admission/discharge policy. Are we being over cautious?

R E McLaughlin, A Stewart Emergency Medicine, Royal Victoria Hospital Grosvenor Road, Belfast BT12 6BA, N Ireland

\section{References}

1 Makwana M, Evans HM, Moore D, et al. Two cases of near asphyxiation in children using non-releasing plastic garden ties. Emerg Med J 2001:18:229-30

2 Stanley RB, Hanson DG. Manual strangulation injuries to the larynx. Arch Otolaryngol 1982;109:44-7.

3 Kuriloff DB, Pincus RL. Delayed airway obstruction and neck abscess following manual strangulation injury. Ann Otol Rhinol Laryngol 1989;98:824-7.

4 Baldwin GA, Macnab AJ, McCormick AQ. Visual loss following traumatic asphyxia in children. J Trauma Injury Infect Crit Care 1988;28:557-8

\section{Author's reply}

We agree entirely, the experience with asphyxiation in children is limited and therefore there is no evidence base as to what is the most appropriate admission/discharge policy. At the Birmingham Children's Hospital we are fortunate in being able to observe less sick children in an accident and emergency based observation bay, in case they get delayed respiratory symptoms, and therefore do not need to admit many children to the paediatric wards.

We were interested to note the reference to subtle changes in visual acuity by Baldwin $e$ al. ${ }^{1}$ This suggests it would be wise to consider visual acuity testing a few weeks after such an incident and we would certainly look towards arranging ophthalmological follow up with these patients in the future.

N Makwana

Royal Wolverhampton NHS Trust, Wolverhampton

\section{Reference}

1 Baldwin GA, Macnab AJ, McCormick AQ Visual loss following traumatic asphyxia in children. J Trauma Injury Infect Crit Care 1988;28:557-8.

\section{Tuberculous osteomyelitis}

Yuen and Tung describe a case of tuberculous osteomyelitis of the foot ${ }^{1}$ and the potential difficulties in making the diagnosis. The authors were fortunate enough to have typical histological biopsy findings that subsequently cultured Mycobacterium tuberculosis (TB), providing diagnostic confirmation and estimations of sensitivities. However, in many instances, the diagnosis of tuberculosis is difficult to verify. For instance, acid fast bacilli may not be identified on biopsy or may be non-tuberculous in origin. Additionally, subsequent culture confirmation can take several weeks or may fail completely, because of the fastidious nature of TB

Although the reliance on clinical suspicion is the basis for the diagnosis of many cases of $\mathrm{TB}$, definitive confirmation is desirable in view of the long term nature of treatment. It is also important to ensure that the organism is not resistant to the chemotherapeutic regimen being used, particularly with the increasing incidence of multidrug resistant TB strains. A number of novel diagnostic techniques have been developed to facilitate this. The use of the polymerase chain reaction to amplify specific TB DNA sequences permits a rapid confirmation of the diagnosis and an estimation of drug sensitivity. ${ }^{2}$ These techniques have been successfully used on both clinical specimens and culture material. ${ }^{3}$ Thus, acid fast bacilli can rapidly be identified as Mycobacterium tuberculosis and an estimation of rifampicin sensitivity can be obtained in a matter of days, free from the contraints of waiting up to several weeks for the standard culture to grow. These techniques should therefore be considered, particularly if the clinical findings are subtle or atypical.

T B L Ho

Infectious Diseases and Microbiology Department, Imperial College of Medicine, Norfolk Place, London W2 1PG; t.ho@ic.ac.uk

\section{References}

1 Yuen MC, Tung WK. An uncommon cause of foot ulcer: tuberculous osteomyelitis. Emerg Med J 2001;18:140-1.

2 Telenti A, Imboden P, Marchesi F, et at. Detection of rifampicin-resistance mutations in Mycobacterium tuberculosis. Lancet 1993;341:647-50

3 Shah S, Miller A, Mastellone A, et al. Rapid diagnosis of tuberculosis in various biopsy and body fluid specimens by the AMPLICOR Mycobacterium tuberculosis polymerase chain reaction test. Chest 1998;113:1190-4.

\section{Authors' reply}

We thank Dr Ho for his comment on our article reporting a young patient with tuberculous osteomyelitis. ${ }^{1}$ We wrote the article from the perspective of emergency medicine. Although polymerase chain reaction (PCR) is a good adjunct to microbiological culture for diagnosing mycobacterium tuberculosis, it is not available to the majority of emergency physicians in Hong Kong. None the less, we should discuss it briefly so that our article is more informative to readers.

Without argument, PCR provides an opportunity for early diagnosis and treatment. However, we should also note the limitation of the PCR especially when the PCR result is negative.

In 1998 Shah et al reported the accuracy of the AMPLICOR PCR test in diagnosing mycobacterium tuberculosis in tissue and body fluid specimens. ${ }^{2}$ In this study, culture proof was adopted as the gold standard for diagnosing tuberculosis. Although 1032 patients were included in this study, only 34 specimens were positive for tuberculosis. Therefore, the sample size was too small and the $95 \%$ confidence interval of the sensitivity was too wide to suggest that PCR would not miss the diagnosis of mycobacterium tuberculosis. In this study, the PCR had a sensitivity of $76.4 \%$, a specificity of 99.8\% when results were compared with the gold standard. With the high specificity, PCR is a good "rule in" test. However, PCR should not be used as a "rule out" test because of the high false negative rate.

In 2000 Lim et al reported the accuracy of the AMPLICOR PCR test in diagnosing pulmonary tuberculosis in smear negative respiratory tract specimens. Once again, the PCR test had a low sensitivity of $44 \%$ and a high specificity of $99 \%$.

With evidence from both studies, a positive PCR test result facilitates early diagnosis, but negative PCR test result cannot exclude mycobacterium tuberculosis. At the moment microbiological culture remains the gold standard for diagnosing tuberculosis and a high index of suspicion for tuberculosis is the key to diagnosis.

M C Yuen, W K Tung Accident and Emergency Department, Kwong Wah Hospital, 25 Waterloo Road, Kowloon, Hong Kong

\section{References}

1 Yuen MC, Tung WK. An uncommon cause of foot ulcer: tuberculous osteomyelitis. Emerg Med J 2001:18:140-1.

2 Shah S, Miller A, Mastellone A, et al. Rapid diagnosis of tuberculosis in various biopsy and body fluid specimens by the AMPLICOR mycobacterium tuberculosis polymerase chain reaction test. Chest 1998:113:1190-4.

3 Lim TK, Gough A, Chin NK, et al. Relationship between estimated pretes probability and accuracy of automated mycobacterium tuberculosis assay in smear-negative pulmonary tuberculosis. Chest 2000;118:641-7.

\section{Journal clubs in clinical medicine}

Journal clubs in clinical medicine have long been recognised as a useful tool for keeping up to date with new developments. ${ }^{1}$ More recently they have been used as a tool for the teaching of critical appraisal, ${ }^{2}$ which for emergency medicine trainees in the UK is an important part of their final fellowship examination.

Since the inception of our journal $\mathrm{club}^{3}$ we have noticed a subtle change in both the quality and quantity of papers in the journals that we chose to review. This made it more difficult to combine both the educational value of critical appraisal and keeping up to date with the relevant advances in our specialty so that we can apply this to our practice of evidence based medicine.

To address this we undertook to review our choice of journals to try to increase our yield of relevant articles. After finding a complete journal list from Medline a consensus opinion was reached on the basis of relevance to practice, past experience of quality of papers, and personal choice. The number of times per year that the journals, or groups of journals, are reviewed depends on the number of issues per year and the likelihood of finding papers relevant to emergency medicine in them.

The complete list of journals and their review rates is shown in table 1 .

We believe that all departments with a journal club should regularly revise their selection of journals in order to increase the value of this important educational process.

$S$ R Jones, M M Harrison,

I W F Crawford, B Ali, E Beattie, S Carley, M Davies, A Ghosh, B Martin, H Paul, R Boyd, K Mackway-Jones, R J Morton Department of Emergency Medicine, Manchester Royal Infirmary, Oxford Road, Manchester M13 9WL, UK

Correspondence to: Professor K Mackway-Jones 